\title{
Longitudinal Associations Between Personality Profile Stability and Adjustment in College Students: Distinguishing Among Overall Stability, Distinctive Stability, and Within-Time Normativeness
}

\author{
Theo A. Klimstra, ${ }^{1}$ Koen Luyckx, ${ }^{2}$ William W. Hale III, ${ }^{1}$ \\ Luc Goossens, ${ }^{2}$ and Wim H. J. Meeus ${ }^{1}$ \\ ${ }^{1}$ Utrecht University, The Netherlands \\ ${ }^{2}$ Catholic University Leuven, Belgium
}

\begin{abstract}
In the present study, longitudinal associations of 3 aspects of personality profile stability (i.e., overall stability, distinctive stability, and within-time normativeness) with 3 adjustment measures (i.e., depressive symptoms, self-esteem, and delinquency) were examined, using 4 waves of longitudinal data on a Belgian college sample $(N=565)$. Longitudinal path models revealed strong longitudinal associations between adjustment and overall stability. Subsequent analyses showed that it is not the degree to which one's personality profile consistently diverges from the average personality profile within a population (i.e., distinctive stability) that is related to adjustment but the degree to which a personality profile of an individual matches the average personality profile within the sample at a certain point in time (i.e., within-time normativeness). The current study thereby underscores the importance of distinguishing normativeness and distinctiveness when examining personality profile stability.
\end{abstract}

Change and stability in personality can be operationalized in several ways (Block \& Robins, 1993). In the past, researchers have examined mean-level changes in personality traits (for an overview, see Roberts, Walton, \& Viechtbauer, 2006), changes in the rank order of individuals on a specific trait (for an overview, see Roberts $\&$ DelVecchio, 2000), and individual differences in changes on traits

Correspondence concerning this article should be addressed to Theo A. Klimstra, Research Centre Adolescent Development, Utrecht University, P.O. Box 80.140, 3508 TC Utrecht, The Netherlands. Email: t.a.klimstra@uu.nl.

Journal of Personality 78:4, August 2010

(C) 2010, Copyright the Authors

Journal compilation (C) 2010, Wiley Periodicals, Inc.

DOI: $10.1111 / \mathrm{j} .1467-6494.2010 .00646 . x$ 
(e.g., Branje, van Lieshout, \& van Aken, 2004; De Fruyt et al., 2006; Roberts, Caspi, \& Moffitt, 2001). All three of these aspects of change focus on changes in individual personality traits. However, these so-called variable-centered approaches tend to ignore that personality is in fact a "peculiar patterning of attributes within the single person" (Allport, 1954, p. 9). Hence, a fourth way to conceptualize change and stability of personality (i.e., personality profile stability) takes Allport's assertion into account by focusing on the stability of a constellation of personality traits within a person across time. Several studies found positive associations between personality profile stability and adjustment (Asendorpf \& van Aken, 1991; Block, 1971; Clausen, 1991; Donnelan, Conger, \& Burzette, 2007; Lönnqvist, Mäkinen, Paunonen, Henriksson, \& Verkasalo, 2008; Ozer \& Gjerde, 1989; Roberts et al., 2001).

However, recent work by Furr (2008) has revealed that profile stability cannot be regarded as a unitary construct. Instead, profile stability should be subdivided into several components: overall stability (i.e., "classic" profile stability), distinctive stability (i.e., the degree to which one's personality profile consistently diverges from the average personality profile within a population), and within-time normativeness (i.e., the degree to which one's personality profile is similar to the average or normative personality profile in a sample at a certain point in time). Until now, it was unclear which component of profile stability drives the effects of overall stability on adjustment. Consequently, the current study examines the longitudinal associations of all three aspects of personality profile stability (i.e., overall stability, distinctive stability, and within-time normativeness) with three adjustment measures (i.e., self-esteem, depressive symptoms, and delinquency). For this purpose, four-wave longitudinal data on a cohort of college students were analyzed.

\section{Personality Profile Stability: Measurement and Links With Adjustment}

Personality profile consistency is usually assessed by measuring profile similarity. For that purpose, an individual needs to be assessed on a set of personality traits on at least two subsequent measurement occasions. A rank-ordered set of personality traits on the first measurement occasion is then correlated with a rank-ordered set of the same personality traits on the second measurement occasion. The 
correlation between these two rank-ordered sets of personality traits, usually measured with $q$-correlations, reflects an individual's personality profile consistency (e.g., Furr, 2008; Roberts et al., 2001). Similar to Pearson test-retest correlations, $q$-correlations can range from -1 to 1 , with a higher $q$-correlation reflecting a more stable personality profile across time.

$Q$-correlations have been examined in several studies on personality development (Asendorpf \& van Aken, 1991; Block, 1971; Clausen, 1991; De Fruyt et al., 2006; Donnelan et al., 2007; Klimstra, Hale, Raaijmakers, Branje, \& Meeus, 2009; Lönnqvist et al., 2008; Ozer \& Gjerde, 1989; Roberts et al., 2001; Robins, Fraley, Roberts, \& Trzesniewski, 2001). These studies found average $q$-correlations ranging from .28 to .85 , with the higher average $q$-correlations found in studies examining personality consistency across shorter time intervals. In addition, two studies (Klimstra et al., 2009; Ozer \& Gjerde, 1989) revealed that $q$-correlations tend to increase with age from childhood to late adolescence. In general, all studies agreed that personality profiles were quite stable across time. Some of the previously mentioned studies (De Fruyt et al., 2006; Donnelan et al., 2007; Robins et al., 2001) have assessed personality consistency with a related index for profile similarity $\left(D^{2}\right)$ alongside $q$-correlations, but these studies merely demonstrated that both indices yielded the same conclusions. Further, $q$-correlations have recently been shown to be among the most reliable measures of profile similarity (McCrae, 2008).

Although the previously mentioned studies found that personality profiles are stable across time in a majority of individuals, they also found substantial interindividual differences in personality consistency (with $q$-correlations across different studies ranging from -.95 to 1.00). For that reason, several studies have set out to investigate the meaning of these interindividual differences in personality consistency by examining associations of $q$-correlations with several adjustment measures. These studies typically found positive associations between personality profile stability on the one hand and desirable personality traits and competence on the other hand (Asendorpf \& van Aken, 1991; Block, 1971; Clausen, 1991; Lönnqvist et al., 2008; Ozer \& Gjerde, 1989). Two other studies (Donnelan et al., 2007; Roberts et al., 2001) obtained similar findings: They found positive associations between profile stability on the one hand and constraint (i.e., traditionalism, harm avoidance, and control) and low levels of negative emotionality on the other hand. 
In sum, several studies have suggested that a stable personality profile is associated with positive adjustment. However, the aforementioned studies have treated personality profile stability as a unitary construct. Recently, Furr (2008) made a case for decomposing profile stability into several elements, which we discuss in the next section.

\section{Unpacking Personality Profile Stability: Overall Stability, Distinctive Stability, and Within-Time Normativeness}

Furr (2008) recently outlined a framework that divides personality profile stability into several components, that is, overall stability, distinctive stability, and within-time normativeness. Overall stability refers to the within-person correlation between a rank-ordered set of traits on Time $t$ and a rank-ordered set of these same traits on Time $t+1$. Thus, overall stability is the type of profile stability that has been utilized in all studies mentioned above. However, when relying on such a global measure of profile stability, researchers are unable to distinguish between distinctive stability and within-time normativeness, which both have been hypothesized to capture different aspects of personality development across time (Furr, 2008).

Distinctive stability reflects the similarity between the distinctive or unique aspects of one's personality at Time $t$ and the distinctive aspects of the personality of that same person at Time $t+1$. More specifically, a person might rate him- or herself higher on Time $t$ Neuroticism and lower on Time $t$ Conscientiousness than the average person in a sample does. If the same person's self-rated trait scores are again above average on Time $t+1$ Neuroticism and below average on Time $t+1$ Conscientiousness, he or she shows a high level of distinctive stability (see Furr, 2008, for a similar example). Therefore, distinctive stability reflects whether an individual diverges from the average person (i.e., the sample norm) in a consistent manner across time.

A third aspect of profile stability is within-time normativeness. Within-time normativeness reflects the correlation between an individuals' personality profile and the average personality profile in a sample (i.e., a personality profile based on sample mean scores on all traits). For instance, if an individual ascribes a higher score to him- or herself on Agreeableness than on Conscientiousness, and the mean of the sample to which he or she belongs is also higher 
for Agreeableness than for Conscientiousness, this individual would show a high level of within-time normativeness. Because within-time normativeness reflects the correlation between an individual's personality profile and the normative profile for a certain sample at a certain point in time, within-time normativeness is captured through the use of a within-time correlation. Overall stability and distinctive stability, on the other hand, reflect an individual's personality consistency across time. As such, only one measurement occasion is needed to calculate within-time normativeness, whereas the calculation of overall stability and distinctive stability requires two measurement occasions.

Furr (2008) stated that the associations between personality profile stability and adjustment found in previous studies could very well be caused by the effect of within-time normativeness on adjustment, which he referred to as the normativeness problem. To deal with this normativeness problem, an approach in which several components of personality profile stability are related to adjustment is recommended. Such an approach would allow for investigating whether it is the cross-temporal stability of a personality profile that is associated with adjustment or, as hypothesized by Furr, whether it is within-time normativeness that causes these associations. As noted, both types of profile stability were not distinguished in previous studies.

\section{Aims of the Current Study}

The current study is the first to empirically decompose personality profile stability into overall stability, a normative component (i.e., within-time normativeness), and a distinctive component (i.e., distinctive stability) and to relate these three components of personality profile stability to adjustment (i.e., self-esteem, depressive symptoms, and delinquency). To infer the directionality of the relations between components of personality profile stability and adjustment, we simultaneously test whether these different components of personality profile stability predict levels of adjustment or whether adjustment predicts these components of personality profile stability. In the current study, the focus is mainly on depressive symptoms as a measure of adjustment, but we also briefly discuss how other indices of adjustment (i.e., self-esteem and delinquency) were related to overall profile stability, distinctive stability, and within-time 
normativeness. Our four-wave longitudinal design allows for examining whether obtained associations are replicated across time.

Based on the findings of the previous studies reviewed above, overall stability is expected to be positively associated with adjustment. The examination of the relations of distinctive stability and within-time normativeness with adjustment is more exploratory in nature because the current study is the first to examine these associations. Thereby, the current study will provide a first attempt to shed light on Furr's (2008) proposition that it is the within-time normativeness component of overall personality profile stability that is responsible for the relations with adjustment that have typically been found in previous studies, and not so much the distinctiveness component.

\section{METHOD}

\section{Participants and Procedure}

Participants were drawn from the Leuven Trajectories of Identity Development Study (L-TIDES; Luyckx, Goossens, \& Soenens, 2006), a seven-wave longitudinal study on college students from the faculty of Psychology and Educational Sciences at a large Belgian university. The first wave was collected at the end of 2002, and the Big Five of personality and the adjustment measures were assessed four times on an annual basis. Consequently, these four waves were used for the present article. At Time 1 , a total of 565 college students $(85.3 \%$ female, $14.7 \%$ male; $M_{\text {age }}=18.63$ years, $\left.S D=.61\right)$ participated. Permission to undertake this study was granted by the Institutional Review Board within the researchers' department. Participants signed a standard consent form before participating in the first wave of data collection. During the consent process, participants were informed that they could refuse or discontinue participation at any time. All students were assigned a unique code number to ensure confidentiality.

As in many longitudinal studies, data were missing at different time points for different participants. To minimize the bias associated with attrition and missing data, we used the expectation maximization (EM) algorithm to impute missing data (Little \& Rubin, 1987; Schafer \& Graham, 2002). Only individuals who participated in at least two of four measurement waves $(N=485)$ were included. Overall, $12.31 \%$ of the data was missing. Participants with and without complete data were compared using Little's (1988) Missing Completely At Random (MCAR) test. This yielded a nonsignificant chi-square value $\left(\chi^{2}(120)=8.92, n s\right)$, suggesting that missing values could be reliably estimated. 


\section{Measures}

\section{Personality}

As a measure of Costa and McCrae's (1992) Five Factor Model of personality, participants completed the Dutch version of the well-established 60-item NEO Five-Factor Inventory (NEO-FFI; Hoekstra, Ormel, \& De Fruyt, 1996). Cronbach's alphas across waves ranged from .87 to .89 for Neuroticism, from .80 to .82 for Extraversion, from .70 to .75 for Openness, from .68 to .72 for Agreeableness, and from .79 to .84 for Conscientiousness.

\section{Depressive Symptoms}

We used a 12-item shortened version of the Center for Epidemiologic Studies Depression Scale (CES-D; Radloff, 1977) to assess depressive symptoms. Items were scored on a 4-point Likert-type rating scale (ranging from seldom to most of the time or always) and refer to cognitive, somatic, and psychological symptoms of depression. Participants were asked to indicate how often they experienced these symptoms during the past week. A sample item is "During the last week, I felt depressed." Cronbach's alphas across waves ranged from .88 to .89 .

\section{Self-Esteem}

General self-esteem was measured with the Rosenberg Self-Esteem Scale (RSES; Rosenberg, 1965). A sample item is "I feel that I have a number of good qualities." This scale contains 10 items scored on a 4-point Likerttype rating scale (ranging from does not apply to me at all to applies to me very well). Cronbach's alphas across waves ranged from .91 to .92 .

\section{Delinquency}

Delinquency was assessed with 11 items adapted from the Deviant Behavior Scale (DBS; Weinmann, 1992). Participants were asked to indicate on a 5point Likert-type rating scale (ranging from does not apply to me at all to applies to me very well) the degree to which they engaged in deviant behaviors (e.g., shoplifting and destroying other people's property) during the past 6 months. Cronbach's alphas across waves ranged from .63 to .76 .

\section{Strategy of Analyses}

As a first step, overall personality stability was calculated with $q$-correlations. A $q$-correlation was calculated for each individual sepa- 
rately, by correlating a rank-ordered set of Big Five traits on one measurement occasion (e.g., T1) with a rank-ordered set of the same Big Five traits on the subsequent measurement occasion (e.g., T2). Because there were four annual measurement waves of Big Five data available in the current study, this procedure resulted in three $q$-correlations of overall stability (i.e., for T1-T2, T2-T3, and T3-T4) for each individual.

Second, distinctive stability was calculated. This required us to center scores of all individuals on every Big Five dimension on all measurement waves (Furr, 2008). That is, we subtracted the mean scores from the raw scores for each individual on every measurement occasion. Distinctive similarity was calculated with $q$-correlations by correlating a rankordered set of centered Big Five traits on one measurement occasion (e.g., T1) with a rank-ordered set of the same Big Five traits (which were again centered) on the subsequent measurement occasion (e.g., T2). Because there were four annual measurement waves of Big Five data available, we were able to calculate three $q$-correlations of distinctive stability (i.e., T1-T2, T2-T3, T3-T4) for each individual.

Third, we calculated within-time normativeness coefficients for each individual on every measurement occasion. For that purpose, we first calculated sample mean scores for each Big Five trait on every measurement occasion. As a next step, we calculated within-time normativeness with $q$ correlations by correlating a rank-ordered set of the sample means on all Big Five traits with a rank-ordered set of Big Five traits for an individual. We followed this procedure for every person in the sample and repeated it for each measurement occasion. As within-time normativeness coefficients represent within-time correlations, there were four measurements of within-time normativeness available (i.e., T1, T2, T3, and T4 within-time normativeness).

Longitudinal associations between adjustment and all three facets of Big Five profile stability were assessed with longitudinal path analyses by means of structural equation modeling in Mplus 4.2 (Muthén \& Muthén, 2007). Model fit was judged by assessing root-mean-square error approximations (RMSEAs), comparative fit indices (CFIs), and Tucker Lewis Indices (TLIs).

RMSEAs below .08 and CFIs and TLIs over .95 indicate adequate model fit; relatively lower RMSEAs and higher CFIs and TLIs indicate a better fit when comparing models (Kline, 1998). Further, we used chisquare difference tests to compare model fits of nested models. To test whether stability paths of adjustment differed from those of the profile stability measures, we first constrained the stability paths of adjustment measures to be equal across waves and constrained the stability paths of a profile stability measure to be equal across waves. In a subsequent step, we used chi-square difference tests to assess whether stability paths of adjust- 
ment differed from those of the profile stability measure. We used a similar procedure to test whether cross paths from adjustment to a profile stability measure differed from those of the profile stability measure to adjustment.

\section{RESULTS}

Overall stability, distinctive stability, and within-time normativeness were calculated for each individual. Descriptive statistics for these three aspects of profile stability and depressive symptoms on the various measurement waves are provided in Table 1.

To test the longitudinal associations of overall stability, distinctive stability, and within-time normativeness with depressive symptoms, we tested longitudinal path models. ${ }^{1,2}$ We first discuss the longitudinal associations between overall stability and depressive symptoms. Next, we move on to examine how the two components underlying overall profile stability (i.e., distinctive stability and within-time normativeness) are related to depressive symptoms.

\section{Overall Profile Stability}

The models that were used to examine relations between depressive symptoms and overall profile stability included all stability paths (e.g., T1 depressive symptoms $\rightarrow \mathrm{T} 2$ depressive symptoms, T1-T2 overall profile stability $\rightarrow \mathrm{T} 2-\mathrm{T} 3$ overall profile stability) and cross paths (e.g., T1 depressive symptoms $\rightarrow$ T1-T2 overall profile stability). In the final model (see Figure 1), stability paths for depressive symptoms and overall profile stability, as well as cross paths from overall profile stability to depressive symptoms, were freely estimated. Cross paths from depressive symptoms to overall personality

1. We first tested models including only main effects of Big Five profile consistency on adjustment and models including main effects of adjustment on Big Five profile consistency. Because these models yielded the exact same conclusions as the reciprocal models, we present only the reciprocal models.

2. To improve model fit, one extra stability path was added for Big Five profile consistency (i.e., T1-T2 Big Five profile consistency $\rightarrow$ T3-T4 Big Five profile consistency) in each of the three models that are discussed. For adjustment, we added three extra stability paths (i.e., T1 adjustment $\rightarrow$ T3 adjustment; T1 adjustment $\rightarrow$ T4 adjustment; T2 adjustment $\rightarrow$ T4 adjustment) to improve model fit. A similar procedure was used in the models concerning distinctive stability. The same procedure has also been used in previous studies (e.g., Cui, Donnelan, \& Conger, 2007). 


\section{Table 1}

Means and Standard Deviations of Depressive Symptoms and Three Components of Profile Stability

\begin{tabular}{|c|c|c|c|c|}
\hline & $\mathrm{T} 1$ & $\mathrm{~T} 2$ & $\mathrm{~T} 3$ & $\mathrm{~T} 4$ \\
\hline Measure & $M(S D)$ & $M(S D)$ & $M(S D)$ & $M(S D)$ \\
\hline \multicolumn{5}{|l|}{ Adjustment } \\
\hline Depressive symptoms & $1.80(.51)$ & $1.72(.50)$ & $1.73(.47)$ & $1.70(.43)$ \\
\hline \multicolumn{5}{|l|}{ Profile stability } \\
\hline Overall stability & $.72(.34)$ & $.74(.35)$ & $.76(.36)$ & \\
\hline Distinctive stability & $.59(.43)$ & $.62(.42)$ & $.62(.45)$ & \\
\hline Within-time normativeness & $.54(.49)$ & $.53(.50)$ & $.58(.49)$ & $.68(.44)$ \\
\hline
\end{tabular}

Note. Overall stability and distinctive stability are indexed by $q$-correlations between each pair of subsequent measurement waves. Because there were four measurement waves, there were three pairs of subsequent measurement waves and hence three measurement occasions for overall stability and distinctive stability. $\mathrm{T}=$ Time. Correlations between variables can be obtained from the first author upon request.

stability were constrained to be equal across waves, as chi-square difference tests demonstrated that adding such constraints did not affect model fit. The resulting model had an excellent fit to our data, $\chi^{2} \quad(8)=15.19, \quad n s ; \quad$ CFI $=.99 ; \quad$ TLI $=.96 ; \quad$ RMSEA $=.04 \quad(90 \%$ $\mathrm{CI}=.04-.08)$.

Figure 1 shows that both depressive symptoms and overall stability were reasonably stable over time. Chi-square difference tests revealed that stability paths were significantly stronger for depressive symptoms than for overall profile stability $(p<.001)$. In addition, all of the cross paths from depressive symptoms to overall profile stability were significant, indicating that depressive symptoms were a negative predictor of overall profile stability. However, only one cross path from overall profile stability to depressive symptoms reached significance. This cross path (T1-T2 overall profile stability $\rightarrow \mathrm{T} 2$ depressive symptoms) indicated that overall profile stability was a negative predictor of depressive symptoms. Moreover, chi-square difference tests denoted that this cross path did not significantly differ from the cross paths from depressive symptoms to overall profile stability. The other cross paths from overall profile stability to depressive symptoms were significantly weaker than the paths from depressive symptoms to overall profile stability $(p<.001)$. 


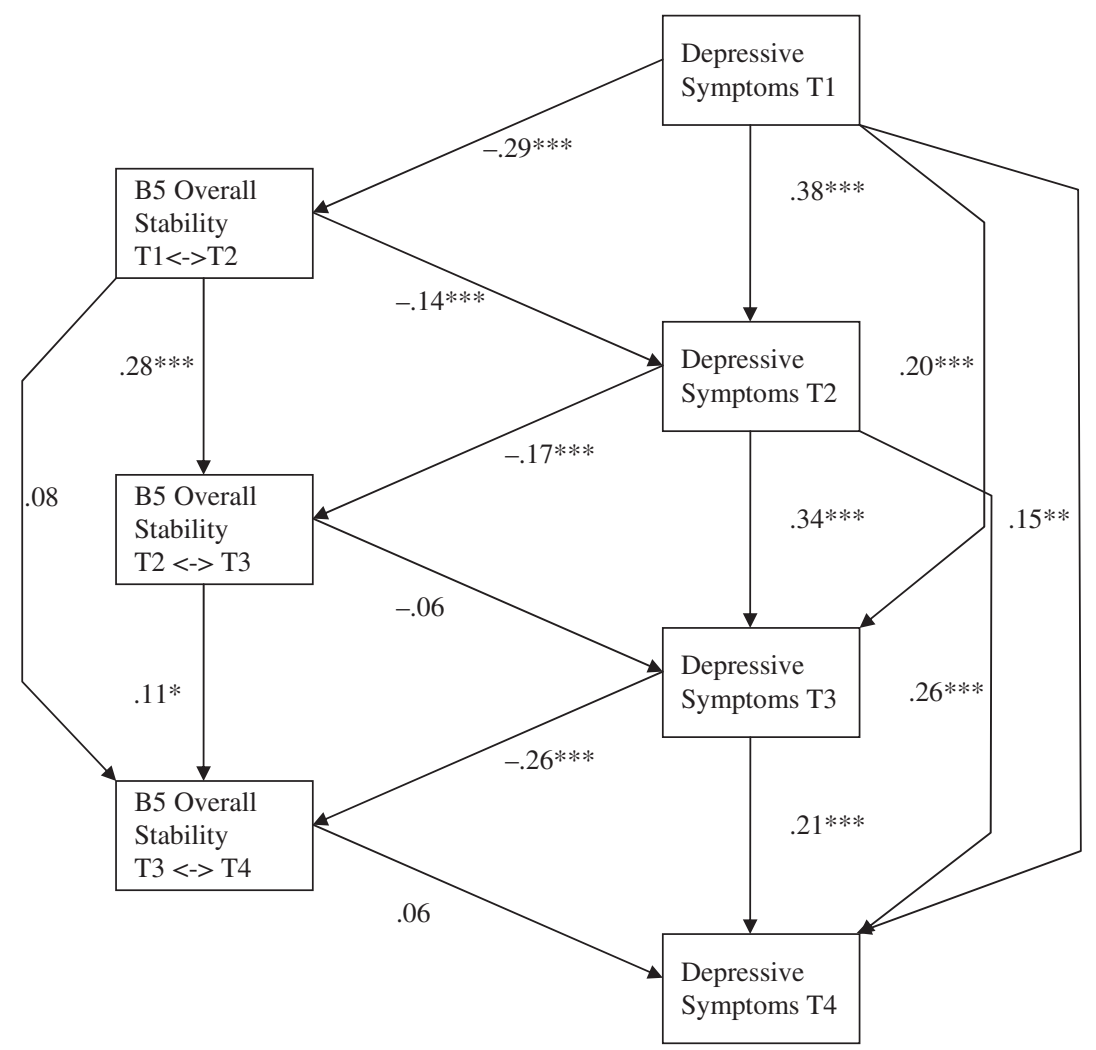

Figure 1

Longitudinal path model for depressive symptoms and overall stability.

\section{Distinctive Profile Stability}

The model relating depressive symptoms to distinctive profile stability was similar to the model relating depressive symptoms to overall profile stability. Stability paths for both variables were freely estimated in this model. All cross paths were constrained to be equal across waves, as adding such constraints did not affect model fit. The resulting model had an exceptional fit, $\chi^{2}(10)=7.13, n s ; \mathrm{CFI}=1.00 ; \mathrm{TLI}=1.02$; RMSEA $=.00(90 \% \mathrm{CI}=.00-.04)$ and is displayed in Figure 2.

Both constructs were reasonably stable across time, but the stability paths of depressive symptoms were significantly stronger than those for distinctive stability $(p<.001)$. None of the cross paths reached significance. 


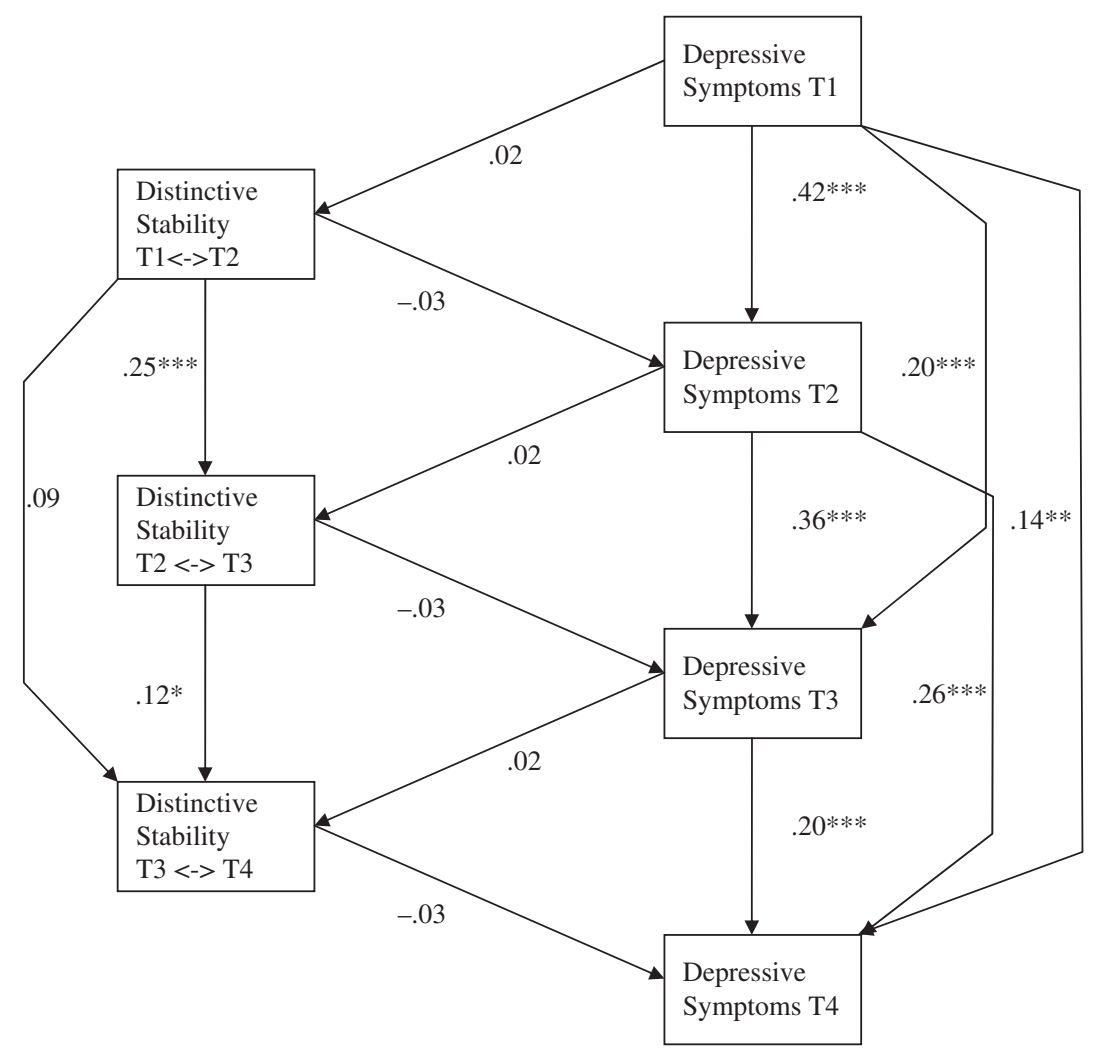

Figure 2

Longitudinal path model for depressive symptoms and distinctive stability.

\section{Within-Time Normativeness}

We used longitudinal path models including stability paths (e.g., T1 depressive symptoms $\rightarrow$ T2 depressive symptoms), within-time correlations (e.g., T1 within-time normativeness $\leftrightarrow \mathrm{T} 1$ depressive symptoms), and cross paths (e.g., T1 depressive symptoms $\rightarrow$ T2 within-time profile normativeness). Stability paths for depressive symptoms were freely estimated, similar to cross paths and correlations between depressive symptoms and within-time personality profile normativeness. Stability paths of within-time normativeness were constrained to be equal across time because adding such constraints did not affect model fit. The resulting model (displayed in Figure 3) 


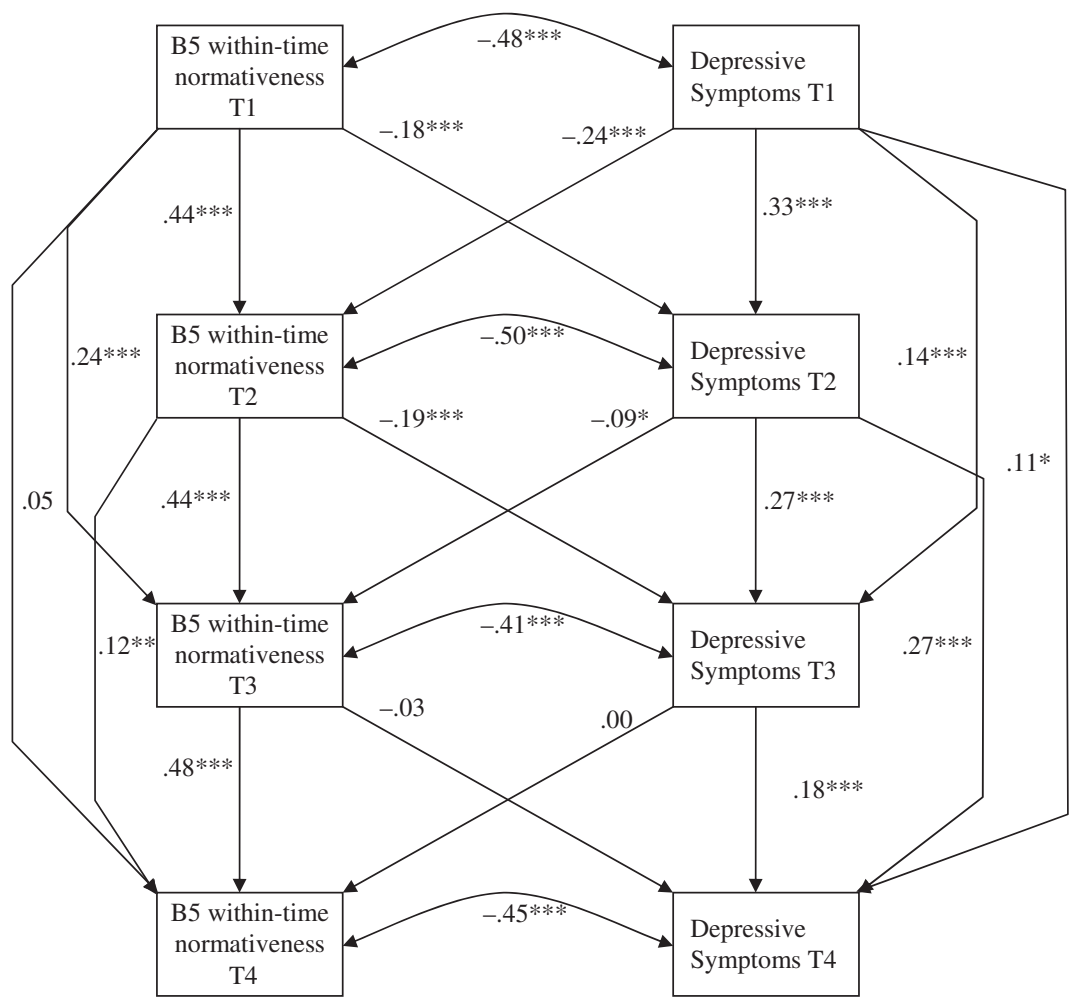

Figure 3

Longitudinal path model for depressive symptoms and within-time normativeness.

had an excellent fit, $\chi^{2}(10)=7.16, n s ; \mathrm{CFI}=1.00 ; \mathrm{TLI}=1.01$; RMSEA $=.00(90 \% \mathrm{CI}=.00-.04)$.

As Figure 3 shows, within-time profile normativeness was more stable across time than depressive symptoms $(p<.001)$. Depressive symptoms and within-time profile normativeness were strongly related to each other at the initial level, and there was also evidence for correlated change. In addition, two out of three cross paths from depressive symptoms to within-time profile normativeness reached significance. Both paths revealed that higher levels of depressive symptoms on one measurement occasion predicted relative decreases of within-time normativeness on the subsequent measurement wave. Two out of three cross paths from within-time normativeness to depressive symptoms reached 
significance. These cross paths indicated that higher levels of within-time normativeness predicted relative decreases in depressive symptoms. Overall, the cross paths from within-time normativeness to depressive symptoms turned out to be equally strong as the cross paths from depressive symptoms to within-time normativeness.

\section{Ancillary Analyses With Self-Esteem and Delinquency}

To examine whether our findings could be generalized across a wider range of adjustment measures, we ran a series of models to test the longitudinal associations between self-esteem and delinquency on the one hand and overall stability, distinctive stability, and withintime normativeness on the other hand. The longitudinal associations obtained with these models are depicted in Table 2. To facilitate comparisons with the depression models, the standardized estimates of those models are also shown in Table 2.

As shown in Table 2, our analyses with self-esteem and delinquency as indicators of adjustment produced similar results as our analyses with depressive symptoms did. That is, adjustment (indicated by higher levels of self-esteem and lower levels of delinquency) was positively related to overall stability. Similar to our results for depressive symptoms, self-esteem and delinquency were almost unrelated to distinctive stability, while both were strongly associated with within-time normativeness. More specifically, within-time normativeness was negatively related to delinquency and positively associated with self-esteem.

\section{DISCUSSION}

The main purpose of the present study was to examine which component of personality profile stability is responsible for the commonly reported associations with adjustment (Asendorpf \& van Aken, 1991; Block, 1971; Clausen, 1991; Donnelan et al., 2007; Lönnqvist et al., 2008; Ozer \& Gjerde, 1989; Roberts et al., 2001). To achieve this objective, we examined longitudinal associations of three components of personality profile stability (i.e., overall stability, distinctive stability, and within-time normativeness; Furr, 2008) with three adjustment measures (i.e., self-esteem, depressive symptoms, and delinquency) in a sample of college students. 
Table 2

Longitudinal Associations of Overall Stability, Distinctive Stability, and Within-Time Normativeness With Self-Esteem and Delinquency (Standardized Estimates)

\begin{tabular}{|c|c|c|c|}
\hline & Depression & Self-Esteem & Delinquency \\
\hline \multicolumn{4}{|c|}{ Overall stability: Cross paths } \\
\hline Adj $\mathrm{T} 1 \rightarrow$ OS T1-T2 & $-.29 * * *$ & $.26^{* * * *}$ & $-.14 * * *$ \\
\hline Adj $\mathrm{T} 2 \rightarrow$ OS T2-T3 & $-.17^{* * * *}$ & $.16^{* * * * *}$ & $-.07^{* * * *}$ \\
\hline Adj T3 $\rightarrow$ OS T3-T4 & $-.26^{* * * *}$ & $.24^{* * * *}$ & $-.09 * * * *$ \\
\hline $\mathrm{OS} \mathrm{T} 1-\mathrm{T} 2 \rightarrow$ Adj T2 & $-.14^{* * * *}$ & .04 & .00 \\
\hline OS T2-T3 $\rightarrow$ Adj T3 & -.06 & .01 & .00 \\
\hline OS T3-T4 $\rightarrow$ Adj T4 & -.06 & $-.08 *$ & .00 \\
\hline \multicolumn{4}{|c|}{ Distinctive stability: Cross paths } \\
\hline Adj T1 $\rightarrow$ DS T1-T2 & .02 & $-.06^{*}$ & -.02 \\
\hline Adj T2 $\rightarrow$ DS T2-T3 & .02 & $-.06^{*}$ & -.01 \\
\hline Adj T3 $\rightarrow$ DS T3-T4 & .02 & $-.05^{*}$ & $.10^{*}$ \\
\hline $\mathrm{DS} \mathrm{T} 1-\mathrm{T} 2 \rightarrow$ Adj T2 & -.03 & .00 & $.09 *$ \\
\hline DS T2-T3 $\rightarrow$ Adj T3 & -.03 & .00 & -.02 \\
\hline DS T3-T4 $\rightarrow$ Adj T4 & -.03 & .00 & -.02 \\
\hline \multicolumn{4}{|c|}{ Within-time normativeness: Within-time correlations } \\
\hline Adj T1 $\leftrightarrow \mathrm{WN} \mathrm{T1}$ & $-.48 * * *$ & $.58 * * * *$ & $-.16^{* * * *}$ \\
\hline Adj $\mathrm{T} 2 \leftrightarrow \mathrm{WN} \mathrm{T} 2$ & $-.50 * * * *$ & $.44^{* * * * *}$ & $-.10 * * * *$ \\
\hline Adj T3 $\leftrightarrow$ WN T3 & $-.41 * * * *$ & $.46^{* * * *}$ & $-.14 * * * *$ \\
\hline Adj T4 $\leftrightarrow$ WN T4 & $-.45 * * *$ & $.55^{* * * *}$ & $-.16^{* * * *}$ \\
\hline \multicolumn{4}{|c|}{ Within-time normativeness: Cross paths } \\
\hline Adj $\mathrm{T} 1 \rightarrow \mathrm{WN}$ T2 & $-.24 * * * *$ & $.31^{* * * *}$ & $-.13 * * * *$ \\
\hline Adj $\mathrm{T} 2 \rightarrow \mathrm{WN} \mathrm{T3}$ & $-.09 *$ & $.10 * *$ & $-.10 * * * *$ \\
\hline Adj T3 $\rightarrow$ WN T4 & .00 & $.10^{* * *}$ & $-.10 * * *$ \\
\hline WN T1 $\rightarrow$ Adj T2 & $-.18 * * *$ & .04 & $-.04 *$ \\
\hline WN T2 $\rightarrow$ Adj T3 & $-.19 * * * *$ & $.17^{* * * * *}$ & $-.05^{*}$ \\
\hline $\mathrm{WN} \mathrm{T3} \rightarrow$ Adj T4 & -.03 & .05 & $-.05^{*}$ \\
\hline
\end{tabular}

Note. Adj $=$ Adjustment (i.e., Self-esteem or Delinquency); OS = Overall Stability; $\mathrm{DS}=$ Distinctive Stability; $\mathrm{WN}=$ Within-Time Normativeness; $\mathrm{T}=$ Time. Stability paths were estimated but omitted from this table. All models depicted in this table had an excellent fit (CFIs were all 1.00; RMSEAs ranged from .00 to .03).

Estimates for stability paths, exact model fits, and other details about the models can be obtained from the first author upon request.

$* p<.05 . * * p<.01 . * * * p<.001$. 


\section{Longitudinal Associations of Adjustment With Three Components of Personality Profile Stability}

Our results indicated that self-esteem, depressive symptoms, and delinquency all had strong longitudinal associations with overall Big Five profile stability. Paths from the adjustment measures to overall profile stability were stronger than the paths in the opposite direction. If overall stability is considered to be a unitary construct, the appropriate conclusion would be that higher levels of well-being and lower levels of internalizing and externalizing problem behaviors seem to foster a more consistent personality profile. This conclusion would be in line with Roberts et al. (2001), who noted that maturity is related to changes toward a desirable endpoint and that the likelihood of personality change diminishes as individuals come closer to that endpoint. However, as previously mentioned, Furr (2008) stated that associations between overall stability and adjustment are likely to be confounded with normativity (i.e., the associations of an individual's personality profile with the personality profile of the average person within a sample) and proposed a paradigm in which normativity and distinctive profile stability (i.e., the degree to which one's personality profile diverges from the average personality profile in a consistent manner) were distinguished from one another. In subsequent sets of analyses, we therefore assessed the longitudinal associations of distinctive profile stability and within-time profile normativity with adjustment.

Longitudinal associations between distinctive personality profile stability and adjustment were, at best, weak. Only some marginally significant paths between delinquency and self-esteem on the one hand and distinctive stability on the other hand were found. With respect to depressive symptoms, there were no associations with distinctive profile stability. Therefore, it would appear that the distinctiveness component of overall profile stability could not account for the strong associations of overall profile stability with adjustment.

In line with Furr's (2008) predictions, our analyses showed that the normativeness component of overall profile stability was strongly associated with adjustment. There were strong concurrent associations of all three adjustment measures with within-time normativeness, and changes in adjustment and within-time normativeness were also strongly associated. These associations suggested that increases 
in adjustment (i.e., increases in self-esteem and decreases in depressive symptoms and delinquency) were associated with changes in personality that caused an individual's personality profile to become more similar to the average ("normative") profile within the sample. Adjustment and normativeness of personality profiles were not only associated with one another, but they also predicted each other over time. There were paths not only from adjustment to normativeness, but also from normativeness to adjustment. Two of the three adjustment measures (i.e., self-esteem and delinquency) turned out to be stronger predictors of within-time profile normativeness than within-time profile normativeness was for these adjustment measures. Depressive symptoms and within-time profile normativeness turned out to be equally predictive of one another.

In sum, our analyses revealed that the longitudinal associations between personality profile stability and adjustment that have been reported in previous studies (Asendorpf \& van Aken, 1991; Block, 1971; Clausen, 1991; Donnelan et al., 2007; Lönnqvist et al., 2008; Ozer \& Gjerde, 1989; Roberts et al., 2001) are quite likely to be caused by the effect that within-time normativeness has on adjustment. At least in the present study, distinctive stability and adjustment were mostly unrelated to one another, whereas normativeness of personality profiles and adjustment were strongly related. Thus, previous studies could follow Furr's (2008) recommendations and reanalyze their data by distinguishing among overall profile stability, distinctive profile stability, and within-time normativeness.

Although distinctive stability was almost unrelated to adjustment, it still constitutes an important aspect of personality profile stability. Table 1 showed that distinctive stability is quite high among early adults, ranging from .59 to .62 in the current study. These relatively high figures suggest that relative differences in personality profiles of early adults are rather consistent across time. For that reason, we can conclude that interindividual differences in personality among early adults are not only quite stable with regard to traits (Costa \& McCrae, 1994) but also with regard to profiles. Given these high figures of distinctive stability in early adults, it would be interesting to examine age-related increases in distinctive stability. Age-related increases in overall stability have previously been reported (e.g., Klimstra et al., 2009; Ozer \& Gjerde, 1989), but there have been no studies examining increases in distinctive stability yet. 
Because the effect of overall stability on adjustment appears to be caused by the effect of normativeness on adjustment, Roberts and colleagues' (2001) proposition that maturity is related to changes toward a desirable endpoint and that the likelihood of personality change diminishes as individuals come closer to that endpoint needs to be reformulated with regard to personality profile stability. Based on our results, changes toward maturation (indicated by increases in self-esteem and decreases in depressive symptoms and delinquency) are related to personality changes that reflect increases in the degree to which an individual's personality profile matches the profile of the average person in a population. In more recent work by Roberts et al. (2006), a similar mechanism is described. They noted that young adults (e.g., college students) are faced with increasing pressure to adhere to social role expectations. Individuals who do not comply with these role expectations are likely to face withdrawal of social approval, which, in turn, can lead to negative consequences such as losing a job. If individuals do meet social expectations, they are likely to receive greater levels of acceptance and social support (Roberts et al., 2006). Roberts et al. (2006) described increases in Conscientiousness and emotional Stability as a movement toward greater compliance with social role expectations. Based on the present findings, we propose that an increase in normativity (i.e., the degree to which one's personality profile matches the average personality profile within a sample) could reflect a similar mechanism. As such, it is not surprising that people with a personality profile that matches social norms reflect higher levels of adjustment than individuals with a profile that diverges from these norms.

\section{Strengths and Limitations of the Current Study}

Taken together, our study has several strengths. The main strength is that it is the first study to examine the longitudinal associations of adjustment with three separate components of personality profile stability: overall profile stability, distinctive profile stability, and within-time normativeness (Furr, 2008). A second strength is the inclusion of four measurement occasions. The inclusion of multiple measurement occasions allowed us to demonstrate that associations did not just occur randomly but could consistently be replicated across time. A third strength was our inclusion of three different 
adjustment measures. This allowed us to demonstrate that our findings were not just specific to negative adjustment in the internalizing domain (i.e., depressive symptoms) but also could be generalized to positive adjustment (i.e., self-esteem) in the internalizing domain and negative adjustment in the externalizing (i.e., delinquency) domain.

Notwithstanding the strengths of the current study, several limitations need to be recognized. First, women comprised $85 \%$ of the sample in the current study. Therefore, the generalizability of our results needs to be confirmed with samples with more balanced gender distributions. Second, our sample included only college students. Therefore, it is not clear to what extent our results are descriptive of the relation between personality consistency and adjustment in the general population of young adults. For that reason, future studies should seek to replicate our findings outside the college context.

That we relied solely on self-reported questionnaires can be considered a third limitation. Especially for personality, other-reported questionnaires have been shown to lead to slightly different conclusions in studies on mean-level change when compared to self-report questionnaires (Branje, van Lieshout, \& Gerris, 2007). Although other-reports are not necessarily more accurate than self-reports when judging personality (Funder, 1995), adding other-reports to studies would provide valuable insights as to whether individuals who are judged inconsistent or nonnormative by others judge themselves in a similar way. In addition, such studies could assess whether self-reported and other-reported components of personality profile stability have a differential effect on, or are differentially affected by, adjustment. Therefore, future studies should ideally assess the relations between both self-reported and other-reported personality profile stability components and adjustment in a single longitudinal design.

Despite these potential limitations, the present study provides a substantial contribution to the current knowledge on personality profile stability, as our findings strongly suggest that it is indeed important to follow Furr's (2008) recommendations to distinguish among different components of personality profile stability. We demonstrated that the stability of the distinctive components of one's personality profile is almost unrelated to adjustment, which suggests that it is not this component of overall profile stability that seems to drive the links with adjustment. Instead, the degree to 
which an individual's personality profile matches the personality profile of an average person in a sample is strongly related to adjustment and would therefore appear to be the most likely cause of the associations of overall profile stability and adjustment. More specifically, our results reveal that individuals who display a personality profile that better matches the personality profile of the average person in a population have higher levels of self-esteem and lower levels of depressive symptoms and delinquency.

\section{REFERENCES}

Allport, G. W. (1954). Personality: A psychological interpretation. New York: Henry Holt.

Asendorpf, J. B., \& van Aken, M. A. G. (1991). Correlates of the temporal consistency of personality patterns in childhood. Journal of Personality, 59, 689-703.

Block, J. (1971). Lives through time. Berkeley, CA: Bancroft Books.

Block, J., \& Robins, R. W. (1993). A longitudinal study of consistency and change in self-esteem from early adolescence to early adulthood. Child Development, 64, 909-923.

Branje, S. J. T., van Lieshout, C. F. M., \& Gerris, J. R. M. (2007). Big five personality development in adolescence and adulthood. European Journal of Personality, 21, 45-62.

Branje, S. J. T., van Lieshout, C. F. M., \& van Aken, M. A. G. (2004). Relations between big five personality characteristics and perceived support in adolescents' families. Journal of Personality and Social Psychology, 86, 615-628.

Clausen, J. S. (1991). Adolescent competence and the shaping of the life course. American Journal of Sociology, 96, 805-842.

Costa, P. T., Jr., \& McCrae, R. R. (1992). Revised NEO Personality Inventory (NEO-PI-R) and the Five Factor Inventory (NEO-FFI): Professional manual. Odessa, FL: Psychological Assessment Resources.

Costa, P. T., Jr., \& McCrae, R. R. (1994). Set like plaster? Evidence for the stability of adult personality. In T. F. Heatherton \& J. L. Weinberger (Eds.), Can personality change? (pp. 21-40). Washington, DC: American Psychological Association.

Cui, M., Donnelan, M. B., \& Conger, R. D. (2007). Reciprocal influences between parents' marital problems and adolescent internalizing and externalizing behavior. Developmental Psychology, 43, 1544-1552.

De Fruyt, F., Bartels, M., van Leeuwen, K. G., De Clercq, B., Decuyper, M., \& Mervielde, I. (2006). Five types of personality continuity in childhood and adolescence. Journal of Personality and Social Psychology, 91, 538-552.

Donnelan, M. B., Conger, R. D., \& Burzette, R. G. (2007). Personality development from late adolescence to young adulthood: Differential stability, norma- 
tive maturity, and evidence for the maturity-stability hypothesis. Journal of Personality, 75, 237-264.

Funder, D. C. (1995). On the accuracy of personality judgment: A realistic approach. Psychological Review, 102, 652-670.

Furr, R. M. (2008). A framework for profile similarity: Integrating similarity, normativeness, and distinctiveness. Journal of Personality, 76, 1267-1316.

Hoekstra, H. A., Ormel, J., \& De Fruyt, F. (1996). NEO persoonlijkheids vragenlijsten: NEO-PI-R, NEO-FFI. Handleiding [NEO personality questionnaires: NEO-PI-R and NEO-FFI-manual]. Lisse, The Netherlands: Swets \& Zeitlinger.

Klimstra, T. A., Hale, W. W., III, Raaijmakers, Q. A. W., Branje, S. J. T., \& Meeus, W. H. J. (2009). Maturation of personality in adolescence. Journal of Personality and Social Psychology, 96, 898-912.

Kline, R. B. (1998). Principles and practice of structural equation modeling. London: Guilford Press.

Little, R. J. A. (1988). A test of missing completely at random for multivariate data with missing values. Journal of the American Statistical Association, 83, $1198-1202$.

Little, R. J. A., \& Rubin, D. B. (1987). Statistical analysis with missing data. New York: Wiley.

Lönnqvist, J. E., Mäkinen, S., Paunonen, S. V., Henriksson, M., \& Verkasalo, M. (2008). Psychosocial functioning in young men predicts their personality stability over 15 years. Journal of Research in Personality, 42, 599-621.

Luyckx, K., Goossens, L., \& Soenens, B. (2006). A developmental contextual perspective on identity construction in emerging adulthood: Change dynamics in commitment formation and commitment evaluation. Developmental Psychology, 42, 366-380.

McCrae, R. R. (2008). A note on some measures of profile agreement. Journal of Personality Assessment, 90, 105-109.

Muthén, L. K., \& Muthén, B. O. (2007). Mplus user's guide (4th ed.). Los Angeles: Muthén \& Muthén.

Ozer, D. J., \& Gjerde, P. F. (1989). Patterns of personality consistency and change from childhood through adolescence. Journal of Personality, 57, 483-507.

Radloff, L. S. (1977). The Center for Epidemiologic Studies-Depression Scale: A self-report depression scale for research in the general population. Journal of Applied Psychological Measurement, 1, 385-401.

Roberts, B. W., Caspi, A., \& Moffitt, T. E. (2001). The kids are alright: Growth and stability in personality development from adolescence to adulthood. Journal of Personality and Social Psychology, 81, 670-683.

Roberts, B. W., \& DelVecchio, W. F. (2000). The rank-order consistency of personality traits from childhood to old age: A quantitative review of longitudinal studies. Psychological Bulletin, 126, 3-25.

Roberts, B. W., Walton, K. E., \& Viechtbauer, W. (2006). Patterns of mean-level change in personality traits across the life course: A meta-analysis of longitudinal studies. Psychological Bulletin, 132, 1-25. 
Robins, R. W., Fraley, R. C., Roberts, B. W., \& Trzesniewski, K. H. (2001). A longitudinal study of personality change in young adulthood. Journal of Personality, 69, 617-640.

Rosenberg, M. (1965). Society and the adolescent self-image. Princeton, NJ: Princeton University Press.

Schafer, J. L., \& Graham, J. W. (2002). Missing data: Our view of the state of the art. Psychological Methods, 7, 147-177.

Weinmann, L. L. (1992). Patterns of change in middle adolescent adjustment: The role of relationships with parents and peers. Dissertation Abstracts International, 53, 2565B-2566B (UMI No. DA92-18128). 